\title{
An Introduction to the EFSUMB Guidelines on Interventional Ultrasound (INVUS)
}

Einführung in die EFSUMB Leitlinien zur Interventionellen Sonographie (INVUS)

C. F. Dietrich ${ }^{1,7}$, T. Lorentzen ${ }^{2}$, P. S. Sidhu ${ }^{3}$, C. Jenssen ${ }^{4}$ O. H. Gilja ${ }^{5}$, F. Piscaglia ${ }^{6}$

Affiliations

Affiliation addresses are listed at the end of the article.

Bibliography

Dol http://dx.doi.org/10.1055/ s-0035-1553462

Ultraschall in Med 2015; 36 : 460-463 (c) Georg Thieme Verlag KG Stuttgart - New York · ISSN 0172-4614

\section{Correspondence}

Prof. Dr. med.

\section{Christoph F. Dietrich}

Med. Klinik 2, Caritaskranken-

haus Bad Mergentheim

Uhlandstr. 7

D-97980 Bad Mergentheim

Germany

Tel.: ++49/(0)7931/5822 01/

2200

Fax: ++49/(0)7931/582290

Christoph.dietrich@ckbm.de

\section{Introduction}

\section{$\nabla$}

In 1972, the delegates of 13 European ultrasound societies met in Basel, Switzerland for the formal foundation of the European Federation of Societies for Ultrasound in Medicine and Biology (EFSUMB). Today the federation comprises 29 national societies with almost 20000 members. EFSUMB is the largest society within the World Federation for Ultrasound in Medicine and Biology (WFUMB). The European federation's purpose has always been to promote the exchange of scientific knowledge in the field of ultrasound [1].

\section{EFSUMB guidelines}

Over the last decade EFSUMB has produced a series of guidelines and recommendations [2-8].

\section{General}

The introduction of any new diagnostic or treatment tool into clinical practice has always resulted in debate in the medical literature, both by clinicians and health care managers. This generally follows a pattern. The first phase is characterized by enthusiasm and optimism driven by the authors performing and promoting the new technique often reporting convincing outcomes, suggesting significant improvement over previous techniques in the same field. This author optimism is frequently counteracted by the skepticism of clinicians not directly involved in the technique. The second phase, often occurring many years later, is characterized by a more equilibrate evaluation prompting learned scientific societies to produce appropriate clinical guidelines, in which a consensus general agreement on the advantages and limitations of the technique and its diagnostic accuracy has been reached $[9,10]$.

\section{CEUS}

Contrast-enhanced ultrasound (CEUS) guidelines were first introduced by EFSUMB in 2004 [2]. The CEUS liver guidelines were updated in 2008 [3] and 2012 [5, 11]. The current version was a successful joint WFUMB/EFSUMB venture simultaneously published in Ultraschall in der Medizin/European Journal of Ultrasound (EJU) [5] and Ultrasound in Medicine and Biology (UMB) [11]. The EFSUMB CEUS non-liver guidelines were also recently published and expand the clinical indications for CEUS to almost 30 applications [4]. These guidelines and recommendations provide general advice on the use of all currently clinically available ultrasound contrast agents (UCA). They are intended to create standard protocols for the use and administration of UCA in clinical applications on an international basis and improve the management of patients worldwide.

\section{Elastography}

In addition, the first elastography guidelines worldwide were introduced and published by EFSUMB in 2013 [7, 8]. All EFSUMB guidelines and good clinical practice recommendations are freely available to download from the EFSUMB website (www.efsumb.org).

\section{Atlas}

An image is worth a thousand words, and an image supplemented by an appropriate number of words is the most effective source of information. For this reason, EFSUMB has begun setting up an atlas of images to illustrate the various published clinical recommendations and guidelines, which includes a variety of contrast-enhanced ultrasound and elastographic applications. The published recommendations have printed page restrictions preventing the inclusion of enough clinical images. In the EFSUMB Atlas you can find pictures and video clips illustrating the typical patterns of most of the ultrasound findings described in the guidelines, with the display indexed according to the structure of the guidelines, to allow easy and rapid access directly to your topic of interest. We are confident that visiting these web pages will be fruitful for your professional activity regardless of your particular field of ultrasound application. We also refer to the current published textbook on interventional ultrasound which was also a motivation for more evidence-based recommendations $[12,13]$.

\section{Impact factor}

The successful EFSUMB guidelines published in our official journal "Ultraschall in der Medizin/ European Journal of Ultrasound" have gained widespread attention. The impact factor (IF) of our journal has climbed to an impressive 4.9, which is the highest IF of an interdisciplinary ultrasound journal worldwide.

\section{Interventional ultrasound (INVUS)}

$\nabla$

It is now pertinent to present the EFSUMB guidelines on ultrasound-guided interventions, procedures that have revolutionized everyday clinical practice in recent decades. The advantages of ultrasound-guided interventions include an unsurpassed sharpness of detail and excellent controllability consequent to the real-time visual display, the wide availability of ultrasound equipment, 
and a simple and straightforward practical technique. Endoscopic ultrasound has made "hidden places" accessible for diagnostic and therapeutic interventions. This makes it even more surprising that interventional ultrasound guidelines have not been previously published.

EFSUMB has accessed a wealth of experience from their expert contributors, who present the subject matter as concretely as possible and offer vivid descriptions of practical techniques. A challenge in the process has been to both unify and respect the differences (national and even regional) often existing in the varied methods of performing INVUS procedures. Particular attention has been given to clinical significance (level of evidence and the more practical grade of recommendation).

The guidelines were discussed during an intensive two-day meeting, attended by all the experts.

The guidelines consist of six main parts:

1. Part I: General aspects

2. Part II: Abdominal diagnostic procedures

3. Part III: Abdominal treatment procedures using the transcutaneous approach

4. Part IV: EUS-guided interventions: General aspects and EUSguided sampling

5. Part V: EUS-guided therapeutic interventions

6. Part VI: Ultrasound-guided vascular interventions

\section{Methodology}

The INVUS guidelines were commissioned by the executive committee of EFSUMB. A steering committee was then appointed with the purpose of defining the disposition and general content of the guidelines, with subsequent invitation of European experts, based on their publications records and expertise in the different fields, to partake in guideline development. Six author subgroups of authors were established with specific responsibility for each section of the INVUS guidelines. Together with the steering committee, the section leaders defined the subchapters and key topics of the 6 guidelines sections. Small groups of 2-3 authors were responsible for each subchapter. A literature search was performed systematically in PubMed using predefined key words and MeSH terms and also manually using reference lists of articles retrieved by systematic search, books and reviews. In principle, the search was restricted to English language papers, in particular guidelines, meta-analyses and systematic reviews, original research articles (randomized controlled trials, prospective studies, retrospective studies, case series $\geq 6$ cases), and results of clinical registries and surveys. English abstracts and single case studies were included only in select circumstances. Evidence tables were generated for each key topic. The Levels of Evidence of the Oxford Centre for Evidence-Based Medicine (March 2009 edition) were used to judge the level of evidence (LoE) and grade of recommendations (GoR) [http://www.cebm.net/oxford-centre-evidence-based-medicinelevels-evidence-march-2009]. Drafts of recommendations and comments were provided by one author, revised by the other members of the small author groups and reviewed by the section leaders. After approval by the subgroup members, the revised drafts were submitted to the whole expert group approximately 2 weeks before the expert meeting, held in Frankfurt, Germany on January 30 - 31, 2015 (๑ Fig. 1). All recommendations were discussed, improved, and finally subjected to a vote by the expert panel. Consensus was graded using a simple system: strong consensus $=>95 \%$ of experts' votes; broad agreement $=>75-95 \%$ of experts' votes; majority consensus $=>50-75 \%$ of experts' votes; dissent $=<50 \%$ of experts' votes in favor of the respective recommendation. Only recommendations with strong consensus or broad agreement were included in the final version of the guidelines. Following the consensus meeting, comments were adapted to the final recommendations and shortened. LoE and GoR were checked by the authors and subgroup leaders. Finally, external experts not involved in the development of the INVUS guidelines reviewed the final drafts with a special focus on LoE and GoR.

It is worth mentioning that, not surprisingly, new and often valid methods are not always or rarely supported by the highest ranking level of scientific evidence, namely randomized controlled trials. Often these innovations are so obviously of benefit that randomized controlled trials involving older, less safe or less effective techniques could be deemed unethical. A provocative example of this is that no randomized controlled trial ever demonstrated that surgical resection of an early stage colon cancer provides longer survival than best supportive care, but nobody questions the use of surgery. In other settings, the question could be raised as to the relevance of setting up a costly randomized controlled trial, e.g. for the comparison of similar but not identical types of needles. Thus, for many important recommendations reported in these guidelines, the strength of evidence may appear relatively weak in comparison to the value of the recommendation based on clinical expert judgement.

\section{Part I: General aspects}

This part deals with generalities that are important and relevant for all kind of INVUS procedures, diagnostic as well as therapeutic: B-mode imaging and the use of CEUS in INVUS procedures; guiding techniques including fusion imaging; patient information, informed consent, and patient preparation; local anesthesia and sedation; hygiene management; puncture routes and accessing techniques; how to reduce and/or eliminate complications; and finally how INVUS is organized locally.

\section{Part Il: Abdominal diagnostic procedures}

This part deals with the workup both in clinical terms and in imaging, prior to the use of an interventional procedure to either diagnose or treat an abnormality. The section is divided into an organ-specific discussion and particular targeted 'niche' areas that will concern readers of the guidelines. All imaging modalities play a role in the workup of these patients, and an ultrasoundguided procedure will not always be the most appropriate imaging tool. This is clearly detailed with evidence-based assessment of the diagnostic route and the final image approach to resolve the clinical situation.

\section{Part III: Abdominal treatment procedures (using the transcutaneous approach)}

Therapeutic abscess drainage and the drainage of pancreatic pseudocysts, interventional tumor ablation techniques, interventional treatments for cysts in general and specifically also parasitic diseases (PAIR for echinococcosis) enrich our daily practice as do established therapeutic procedures such as PTCD, PEG, PUG, biliary and urinary bladder drainage and nephrostomy. In the matter of interventional guidance and approach, it is often necessary to decide between the competing modalities of CT guidance and other imaging techniques, which in some cases can and should be used to complement or supplement one another. The role of ultrasound contrast agents in the preparation, support and guidance of interventional procedures is also addressed. 


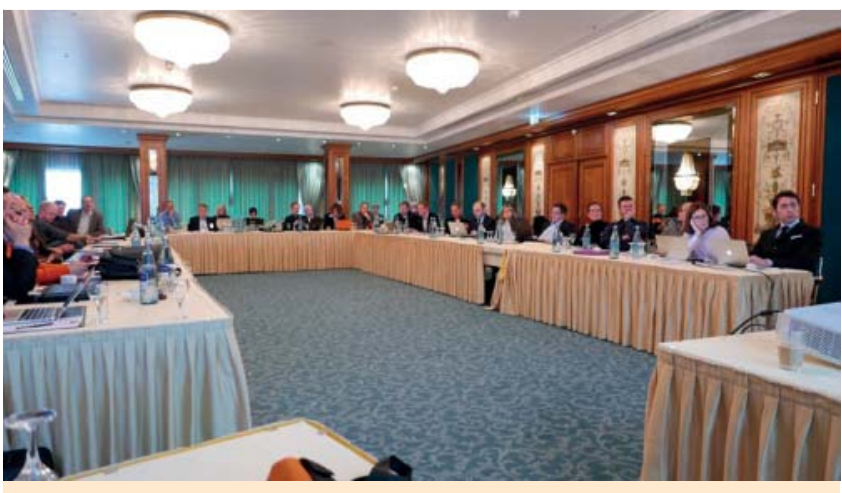

Fig. 1 The guidelines were discussed during an intensive two-day meeting, attended by all the experts.

Symptom-oriented palliative care interventions are an important issue discussed at the end of the chapter.

\section{Part IV: EUS-guided interventions: General aspects and} EUS-guided sampling

EUS-guided sampling was introduced in the early $1990 \mathrm{~s}$ and 20 years later was proclaimed to have afforded a "disruptive innovation effect" for pancreatic pathology [14]. It combines the most advanced high-resolution ultrasound imaging of lesions within the wall and in the vicinity of the gastrointestinal tract and provides safe and effective tissue acquisition. The guidelines deal with the indications and clinical impact of EUS-guided sampling and try to balance advantages and drawbacks in comparison with image-guided percutaneous biopsy. Needle choice and biopsy technique as well as specimen processing are crucial for success, and therefore evidence-based recommendations are given for almost all steps of EUS-guided sampling as well as for safe performance.

\section{Part V: EUS-guided therapeutic interventions}

Soon after the introduction of EUS-guided fine-needle aspiration, the therapeutic potential of EUS guidance was discovered, and the first successful attempts at EUS-guided celiac plexus neurolysis, pseudocyst drainage, and cholangiopancreatography were reported. The spectrum of EUS-guided therapeutic procedures has broadened since those first steps, and scientific literature on EUS-guided treatment is prospering. This is reflected by evidence-based recommendations on EUS-guidance of tumor ablation therapy, injection treatment of the celiac plexus, vascular interventions, drainage of pancreatic and non-pancreatic fluid collections and - last but not least - drainage procedures of the biliary tree and pancreatic duct. EUS-guided therapeutic techniques are compared with their percutaneous alternatives to help the clinician to choose the most appropriate solutions for challenging therapeutic problems.

\section{Part VI: US-guided vascular interventions}

Real-time US guidance for central venous access was performed for the first time in 1986 [15] and, supported by overwhelming evidence, is now regarded as a key safety measure in modern medicine. Systematic analysis of scientific literature shows that US guidance may also facilitate efficacy and reduce adverse events in peripheral venous access and endovascular interventions. Moreover, recommendations endorse the use of ultrasound to detect complications of vascular access and US-guided treatment of arterial pseudoaneurysms.

\section{Online and in-print versions of the guidelines}

The INVUS guidelines will be published in EJU in a detailed online-only version followed by a condensed printed version. Furthermore, some chapters and multimedia material are also intended only for the EFSUMB website. Online versions will help provide more details that do not fit in the limited print space, which will focus on the main issues supporting the recommendations. Revision of the INVUS guidelines is intended in 5 years.

All contributors signed a form to declare any potential conflicts of interest with the guidelines. Financial interest information can be obtained on request from EFSUMB.

\section{Conclusion}

$\nabla$

The guidelines are an expression of interdisciplinary and multiprofessional viewpoints, some of which represent different approaches. This reflects the reality in all its diversity. The principle of "do no harm" is expressed in repeated pleas to apply the techniques judiciously in everyday practice and not to become fascinated with technology for its own sake. The decision to proceed with an interventional procedure is always an individual one and should be made on the basis of its benefit for the patient [12].

\section{Affiliations}

1 Department of Internal Medicine 2, Caritas Krankenhaus, Bad Mergentheim, Germany

2 Department of Gastroenterology, Surgical Section, Herlev Hospital, University of Copenhagen, Herlev, Denmark

3 Department of Radiology, King's College Hospital, London, UK

${ }^{4}$ Department of Internal Medicine, Krankenhaus Märkisch Oderland, Strausberg/Wriezen, Germany

5 National Centre for Ultrasound in Gastroenterology, Department of Medicine, Haukeland University Hospital, Bergen, Norway and Department of Clinical Medicine, University of Bergen, Norway

${ }^{6}$ Unit of Internal Medicine, Dept of Medical and Surgical Sciences, University of Bologna Hospital S.Orsola Malpighi, Bologna, Italy

7 Sino-German Research Center of Ultrasound in Medicine, The First Affiliated Hospital of Zhengzhou University, Zhengzhou, China

\section{Acknowledgments \\ $\nabla$}

EFSUMB is a registered UK charity and is funded by subscriptions from its member national societies to carry out educational and scientific activities as detailed in the constitution of the society. These guidelines were made possible by the generous support of the following companies listed in alphabetical order: Bracco, Covidien, Esaote, Fujifilm, GE Healthcare, Hitachi, Olympus, Peter Pflugbeil, Philips, Siemens, Supersonic and Toshiba.

EFSUMB only accepts donations from industry if unrestricted and for educational purposes and favors working with a consortium of companies. EFSUMB is satisfied that accepting such support did not adversely affect its independent status and/or reputation. There was no attempt on the part of the companies to influence EFSUMB policy or actions explicitly or implicitly and EFSUMB retains full editorial control and copyright over all publications and printed materials funded by these companies. 
The expert opinions expressed within the guidelines are independent and free of bias, based on the best evidence for clinical practice and any potential conflict of interest of each individual author is disclosed in the online version for maximal transparency, as should be expected for guidelines drafted according to modern standards.

\section{References}

1 Dietrich CF, Rudd L. The EFSUMB website, a guide for better understanding. Med Ultrason 2013; 15 (3): 215-223

2 Albrecht T, Blomley M, Bolondi L et al. Guidelines for the use of contrast agents in ultrasound. Ultraschall in Med 2004; 25 (4): 249-256

3 Claudon M, Cosgrove D, Albrecht $T$ et al. Guidelines and good clinical practice recommendations for contrast enhanced ultrasound (CEUS) - update 2008. Ultraschall in Med 2008; 29 (1): 28 - 44

4 Piscaglia F, Nolsoe C, Dietrich CF et al. The EFSUMB Guidelines and Recommendations on the Clinical Practice of Contrast Enhanced Ultrasound (CEUS): update 2011 on non-hepatic applications. Ultraschall in Med 2012; 33 (1): $33-59$

5 Claudon M, Dietrich CF, Choi BI et al. Guidelines and good clinical practice recommendations for Contrast Enhanced Ultrasound (CEUS) in the liver - update 2012: A WFUMB-EFSUMB initiative in cooperation with representatives of AFSUMB, AIUM, ASUM, FLAUS and ICUS. Ultraschall in Med 2013; 34 (1): 11-29

6 Dietrich CF, Averkiou MA, Correas JM et al. An EFSUMB introduction into Dynamic Contrast-Enhanced Ultrasound (DCE-US) for quantification of tumour perfusion. Ultraschall in Med 2012; 33 (4): 344- 351
7 Bamber J, Cosgrove D, Dietrich CF et al. EFSUMB guidelines and recommendations on the clinical use of ultrasound elastography. Part 1: Basic principles and technology. Ultraschall in Med 2013; 34 (2): 169 184

8 Cosgrove D, Piscaglia F, Bamber J et al. EFSUMB Guidelines and Recommendations on the Clinical Use of Ultrasound Elastography. Part 2: Clinical Applications. Ultraschall in Med 2013; 34 (3): 238-253

9 Bolondi $L$. The appropriate allocation of CEUS in the diagnostic algorithm of liver lesions: a debated issue. Ultraschall in Med 2013; 34 (1): $8-10$

10 Ingravallo F, Dietrich $\mathrm{CF}$, Gilja $\mathrm{OH}$ et al. Guidelines, clinical practice recommendations, position papers and consensus statements: definition, preparation, role and application. Ultraschall in Med 2014; 35 (5): $395-399$

11 Claudon M, Dietrich CF, Choi BI et al. Guidelines and good clinical practice recommendations for Contrast Enhanced Ultrasound (CEUS) in the liver - update 2012: A WFUMB-EFSUMB initiative in cooperation with representatives of AFSUMB, AIUM, ASUM, FLAUS and ICUS. Ultraschall Med Biol 2013; 39 (2): 187-210

12 Dietrich CF, Nuernberg D. Interventional ultrasound. Stuttgart: Thieme Publisher; 2014

13 Dietrich $C F$, Nuernberg D. Interventionelle Sonographie. Stuttgart: Thieme; 2011

14 Eltoum IA, Alston EA, Roberson J. Trends in pancreatic pathology practice before and after implementation of endoscopic ultrasound-guided fine-needle aspiration: an example of disruptive innovation effect? Arch Pathol Lab Med 2012; 136 (4): 447-453

15 Yonei A, Nonoue T, Sari A. Real-time ultrasonic guidance for percutaneous puncture of the internal jugular vein. Anesthesiology 1986; 64 (6): $830-831$ 